\section{Minimal Projective Reconstruction Including Missing Data}

\author{
Fredrik Kahl, \\ Anders Heyden, Member, \\ IEEE Computer Society, and Long Quan
}

\begin{abstract}
The minimal data necessary for projective reconstruction from image points is well-known when each object point is visible in all images. In this paper we formulate and propose solutions to a new family of reconstruction problems for multiple images from minimal data, where there are missing points in some of the images. The ability to handle the minimal cases with missing data is of great theoretical and practical importance. It is unavoidable to use them to bootstrap robust estimation such as RANSAC and LMS algorithms and optimal estimation such as bundle adjustment. First, we develop a framework to parameterize the multiple view geometry needed to handle the missing data cases. Then, we present a solution to the minimal case of eight points in three images, where one different point is missing in each of the three images. We prove that there are, in general, as many as 11 solutions for this minimal case. Furthermore, all minimal cases with missing data for three and four images are catalogued. Finally, we demonstrate the method on both simulated and real images and show that the algorithms presented in this paper can be used for practical problems.
\end{abstract}

Index Terms-Structure recovery, projective reconstruction, structure from motion, projective geometry, missing data.

\section{INTRODUCTION}

RECONSTRUCTING the 3D scene geometry from a number of images is one common goal in computer vision. Recently, reconstruction methods have been successfully extended to projective reconstruction within an uncalibrated framework [4], [7], [9], [16], [8]. Using image measurements, it is only possible to recover the 3D scene geometry ("structure"), camera poses ("motion"), and internal camera calibration up to an unknown 3D projective transformation [4]. With additional scene, motion, or calibration constraints, it is possible to upgrade the projective reconstruction to a (scaled) Euclidean one. In particular, several methods using constraints on the internal camera calibration (so-called autocalibration methods) have been developed, e.g., [20], [10], [12].

Solving minimal cases to perform reconstruction of $3 \mathrm{D}$ points from their $2 \mathrm{D}$ images is of both theoretical and practical importance, whereby, a minimal case is meant that omission of one point in one image gives an infinite number of solutions. It is common to use the solutions obtained from the minimal cases to either bootstrap a robust estimation algorithm such as RANSAC or LMS schema [6], [19], [22] or an optimal estimation algorithm such as bundle adjustment [1]. The minimal data necessary for projective reconstruction is well-known. In the two-view case, the minimum number of points is 7, cf. Sturm's method [17] reintroduced into computer vision in [5], [4], [13]. For three views, the minimum number of points is 6 , cf. [15], [9]. The solutions to these problems rely on solving a cubic equation and, thus, there are, in general, up to three solutions. It is no coincidence that the number of solutions is equal for the two problems. In fact, they are

- F. Kahl and A. Heyden are with the Centre for Mathematical Sciences, Lund University, Box 118, SE-221 00 Lund, Sweden.

E-mail: \{fredrik, heyden\}@maths.lth.se.

- L. Quan is with INRIA Rhône-Alpes, 655 avenue de l'Europe, 38330 Montbonnot St. Martin, France. E-mail: Long.Quan@inrialpes.fr.

Manuscript received 21 June 2000; revised 21 Dec. 2000; accepted 3 Jan. 2001. Recommended for acceptance by P. Meer.

For information on obtaining reprints of this article, please send e-mail to: tpami@computer.org, and reference IEEECS Log Number 112321. dual to each other in the Carlsson duality sense, cf. [2]. However, these minimal cases do not allow the possibility of missing data. In practice, the missing data cases are frequent. The more images we have, the higher possibility that missing data occur. One typical example is illustrated in Fig. 1 in which no matter how we locate the cameras, one of the eight corners will not be visible. Even more, the missing point changes with the viewing position. Three possible camera positions are indicated in the figure that may result in three different missing corners in the images.

This motivates us to formulate a new family of minimal reconstruction problems containing missing data. First, we will develop a framework for efficiently parameterizing the multiple view geometry. Then, we will concentrate on the typical minimal case of eight points in three images, where one different point is missing in each of the three images. We will show that, not surprisingly, the algorithms for missing data cases are generally more complicated than the nonmissing counterparts. The typical minimal case described above has 11 solutions. Finally, a catalogue of the different minimal cases for three and four images will be provided. Although the algebraic solutions are complicated, we demonstrate that the solutions are stable enough for dealing with real image sequences.

Previous work on reconstruction with missing data has been primarily concentrated on solely handling the redundant data cases. For example, the "hallucination" of Tomasi and Kanade [18], the closure constraints in the projective case of Triggs [21], and in the affine case of Kahl and Heyden [11]. The ability to deal with both a minimal case and missing data is, to our knowledge, new to the computer vision community.

The paper is organized as follows: In Section 2, we first formulate the minimal missing data problem for reconstruction. Then, we solve the minimal case of eight points in three images with one missing point in each image in Section 3. Next, we summarize all known minimal missing data cases for three and four images in a catalogue in Section 4. After that, we evaluate the performance of the solutions on both simulated and real images in Section 5. Finally, some concluding remarks are given in Section 6 .

\section{Problem Formulation}

Throughout the paper, vectors are denoted in lower case boldface and matrices in upper case boldface. Scalars are any plain letters or lower case Greek.

We assume a perspective projection (uncalibrated pin-hole camera) as the camera model. Thus, the object space may be considered as embedded in $\mathcal{P}^{3}$ and the image space embedded in $\mathcal{P}^{2}$. The camera performs a projection from $\mathcal{P}^{3}$ to $\mathcal{P}^{2}$ and can be represented by a $3 \times 4$ matrix $\mathbf{P}_{3 \times 4}$ of rank 3 whose kernel is the projection center. The relation between an object point $\mathrm{x}$ in $\mathcal{P}^{3}$ and an image point $\mathbf{u}$ in $\mathcal{P}^{2}$ can be written

$$
\lambda \mathbf{u}=\mathbf{P}_{3 \times 4} \mathbf{x} .
$$

It is well-known that both the problem of reconstructing six points from three images, see [15], [9], as well as seven points from two images, see [17], [5], have, in general, three different solutions. However, these minimal cases do not allow the possibility of having missing data in any of the images. For practical applications, it is important to be able to deal with the minimal cases which may contain missing data in some of the images. For two images, there are no minimal cases with missing data since a point visible in only one image does not give any constraint on the viewing geometry. So, the minimum number of images in which we are interested is three. 


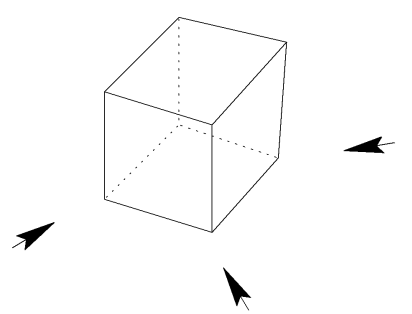

Fig. 1. A frequent practical example of missing data. A cube with eight corners where (in general) only seven corners will be visible in each image. Three possible camera positions resulting in three different missing points are indicated by arrows.

For each point in each image, two quantities are measured $(x-$ and $y$-coordinates). Thus, given six points visible in three images, $6 \cdot 3 \cdot 2=36$ constraints (or equations) are obtained. The unknowns are the $3 \mathrm{D}$ point coordinates and the camera matrices modulo the projective coordinate frame, in total, $3 \cdot 6+3 \cdot 11-15=36$ unknowns. This is a minimal case without missing data as

$$
\text { \#unknowns }=\text { \#equations }=36 .
$$

Starting with the above minimal case, i.e., six points in three images and removing the sixth point from the third image gives, of course, less equations than unknowns. However, trying to add a seventh point visible in images one and three results in $5 \cdot 3 \cdot 2+2 \cdot 2 \cdot 2=38$ (five points in three images and two points in two images) equations and $7 \cdot 3+3 \cdot 11-15=39$ (seven points and three camera matrices up to the projective transformation) unknowns. This is still underdetermined as \#unknowns > \#equations.

By further adding a point in images two and three, we obtain $5 \cdot 3 \cdot 2+3 \cdot 2 \cdot 2=42$ (five points in three images and three points in two images) equations and $8 \cdot 3+3 \cdot 11-15=42$ (eight points and three camera matrices) unknowns, i.e., a minimal case since \#unknowns $=$ \#equations $=42$. This incidence relation can be visualized by the following matrix, where 0 in row $i$ and column $j$ means that point $j$ is missing in image $i$ and $\times$ means visible:

$$
\left[\begin{array}{cccccccc}
\times & \times & \times & \times & \times & \times & \times & 0 \\
\times & \times & \times & \times & \times & \times & 0 & \times \\
\times & \times & \times & \times & \times & 0 & \times & \times
\end{array}\right]
$$

The three images of a cube indicated in Fig. 1 may result in the above incidence relation. We conclude the discussion with a formal problem statement.

Problem 1. Reconstruct the object and the camera matrices (structure and motion) from eight points and three images, where one different point is missing in each image and determine the number of different solutions.

\section{Problem Solution}

In this section, we will first outline a parameterization framework for handling the geometry of multiple cameras. Then, a solution to Problem 1 will be derived. As we are working within an uncalibrated projective setting, all quantities are determined only up to an unknown projective transformation, therefore, we may, without restriction, ${ }^{1}$ introduce a projective coordinate system such that the first five points in space are assigned to the canonical projective coordinates

1. We are implicitly assuming that the first five object points are projectively independent as well as the first four points in each image.

$$
\left[\begin{array}{lllll}
\mathbf{x}_{1} & \mathbf{x}_{2} & \mathbf{x}_{3} & \mathbf{x}_{4} & \mathbf{x}_{5}
\end{array}\right]=\left[\begin{array}{ccccc}
1 & 0 & 0 & 0 & 1 \\
0 & 1 & 0 & 0 & 1 \\
0 & 0 & 1 & 0 & 1 \\
0 & 0 & 0 & 1 & 1
\end{array}\right] .
$$

and the first four image points in each image are assigned to

$$
\left[\begin{array}{llll}
\mathbf{u}_{1} & \mathbf{u}_{2} & \mathbf{u}_{3} & \mathbf{u}_{4}
\end{array}\right]=\left[\begin{array}{cccc}
1 & 0 & 0 & 1 \\
0 & 1 & 0 & 1 \\
0 & 0 & 1 & 1
\end{array}\right] .
$$

Using this choice of coordinates, it is not hard to see that we get the following special form of camera matrices

$$
\mathbf{P}=\left[\begin{array}{llll}
\alpha & 0 & 0 & \delta \\
0 & \beta & 0 & \delta \\
0 & 0 & \gamma & \delta
\end{array}\right]
$$

This reduced camera matrix using canonical projective coordinates has been successfully applied by many researchers [4], [15], [9], [2].

By further using the fact that the fifth basis point in space $(1,1,1,1)$ projects onto the fifth point in each image as $\left(u_{5}, v_{5}, w_{5}\right)$, we obtain

$$
\left\{\begin{aligned}
\lambda_{5} u_{5} & =\alpha+\delta \\
\lambda_{5} v_{5} & =\beta+\delta \\
\lambda_{5} w_{5} & =\gamma+\delta
\end{aligned}\right.
$$

Observe that both the left side and the right side are only determined up to an unknown scale factor. This means that we can fix the scale factors consistently by putting $\lambda_{5}=1$, which gives a natural scale to the camera matrix, inherited from the homogeneous coordinates $\left(u_{5}, v_{5}, w_{5}\right)$. We will assume that this fixation of scales already has been made in the sequel. This leads to the following reduced camera matrix:

$$
\mathbf{P}=\left[\begin{array}{cccc}
u_{5}-\delta & 0 & 0 & \delta \\
0 & v_{5}-\delta & 0 & \delta \\
0 & 0 & w_{5}-\delta & \delta
\end{array}\right]
$$

It is important to observe that this reduced camera matrix only contains one unknown parameter $\delta$. We are now ready to state our main theorem of the section.

Theorem 1. There are, in general, 11 algebraic solutions to Problem 1. The solutions may include complex and not physically feasible solutions.

Proof. Without restrictions, we can make projective changes of coordinates in the object space as well as in the images. Then, we arrive at the reduced camera matrix parameterized by only one unknown $\delta$ as in (3). For a set of three images, we have three unknowns $\delta, \delta^{\prime}$ and $\delta^{\prime \prime}$.

Consider the subconfiguration of six points that are visible in both the first and second image. If the first five points are chosen as a projective basis, then the image of the sixth point in the first image is given by

$$
\lambda_{6}\left[\begin{array}{c}
u_{6} \\
v_{6} \\
w_{6}
\end{array}\right]=\left[\begin{array}{cccc}
u_{5}-\delta & 0 & 0 & \delta \\
0 & v_{5}-\delta & 0 & \delta \\
0 & 0 & w_{5}-\delta & \delta
\end{array}\right]\left[\begin{array}{c}
x_{6} \\
y_{6} \\
z_{6} \\
t_{6}
\end{array}\right],
$$

and, in the second image, by

$$
\lambda_{6}^{\prime}\left[\begin{array}{c}
u_{6}^{\prime} \\
v_{6}^{\prime} \\
w_{6}^{\prime}
\end{array}\right]=\left[\begin{array}{cccc}
u_{5}^{\prime}-\delta^{\prime} & 0 & 0 & \delta^{\prime} \\
0 & v_{5}^{\prime}-\delta^{\prime} & 0 & \delta^{\prime} \\
0 & 0 & w_{5}^{\prime}-\delta^{\prime} & \delta^{\prime}
\end{array}\right]\left[\begin{array}{l}
x_{6} \\
y_{6} \\
z_{6} \\
t_{6}
\end{array}\right] .
$$


Note that they are both homogeneously linear in $x_{6}, y_{6}, z_{6}, t_{6}, \lambda_{6}$, and $\lambda_{6}^{\prime}$ and can be rewritten together as

$$
\left[\begin{array}{cccccc}
u_{5}-\delta & 0 & 0 & \delta & -u_{6} & 0 \\
0 & v_{5}-\delta & 0 & \delta & -v_{6} & 0 \\
0 & 0 & w_{5}-\delta & \delta & -w_{6} & 0 \\
u_{5}^{\prime}-\delta^{\prime} & 0 & 0 & \delta^{\prime} & 0 & -u_{6}^{\prime} \\
0 & v_{5}^{\prime}-\delta^{\prime} & 0 & \delta^{\prime} & 0 & -v_{6}^{\prime} \\
0 & 0 & w_{5}^{\prime}-\delta^{\prime} & \delta^{\prime} & 0 & -w_{6}^{\prime}
\end{array}\right]\left[\begin{array}{c}
x_{6} \\
y_{6} \\
z_{6} \\
t_{6} \\
\lambda_{6} \\
\lambda_{6}^{\prime}
\end{array}\right]=\mathbf{0},
$$

or more compactly denoted as

$$
\mathbf{M}_{6 \times 6}\left(\mathbf{x}_{6}, \lambda_{6}, \lambda_{6}^{\prime}\right)^{T}=\mathbf{0} .
$$

Since the vector $\left(\mathbf{x}_{6}, \lambda_{6}, \lambda_{6}^{\prime}\right)$ does not vanish, the $6 \times 6$ matrix $\mathbf{M}$ has to be singular, i.e., $\operatorname{det} \mathbf{M}=0$. The vanishing of the determinant can be expressed as

$$
\mathbf{u}_{6}^{\prime T} \mathbf{F}_{12} \mathbf{u}_{6}=0,
$$

where $\mathbf{F}_{12}=\left[\operatorname{diag}\left(\mathbf{u}_{5}\right)-\delta \mathbf{I}\right]\left[\delta \mathbf{u}_{5}^{\prime}-\delta^{\prime} \mathbf{u}_{5}\right]_{\times}\left[\operatorname{diag}\left(\mathbf{u}_{5}^{\prime}\right)-\delta^{\prime} \mathbf{I}\right]$ is the fundamental matrix relating image 1 and image 2 . Here, $[\mathbf{u}]_{\times}$ denotes the skew symmetric matrix for the vector $\mathbf{u}$ such that $[\mathbf{u}]_{\times} \mathbf{v}=\mathbf{u} \times \mathbf{v}$, where $\times$ denotes the cross product. Expanding the above equation in the unknowns $\delta, \delta^{\prime}$, results in a polynomial equation $p_{12}\left(\delta, \delta^{\prime}\right)=0$, where

$$
p_{12}=a_{1} \delta^{2} \delta^{\prime}+a_{2} \delta \delta^{\prime 2}+a_{3} \delta^{2}+a_{4} \delta \delta^{\prime}+a_{5} \delta^{2}+a_{6} \delta+a_{7} \delta^{\prime} .
$$

The coefficients are

$$
\begin{aligned}
& a_{1}=\operatorname{det}\left[\mathbf{u}_{6}^{\prime}, \mathbf{u}_{5}^{\prime}, \mathbf{u}_{6}\right], a_{2}=-\operatorname{det}\left[\mathbf{u}_{6}^{\prime}, \mathbf{u}_{5}, \mathbf{u}_{6}\right], \\
& a_{3}=-\operatorname{det}\left[\mathbf{u}_{6}^{\prime}, \mathbf{u}_{5}^{\prime}, \operatorname{diag}\left(\mathbf{u}_{5}^{\prime}\right) \mathbf{u}_{6}\right], \\
& a_{4}=\operatorname{det}\left[\mathbf{u}_{6}^{\prime}, \mathbf{u}_{5}, \operatorname{diag}\left(\mathbf{u}_{5}^{\prime}\right) \mathbf{u}_{6}\right]-\operatorname{det}\left[\operatorname{diag}\left(\mathbf{u}_{5}\right) \mathbf{u}_{6}^{\prime}, \mathbf{u}_{5}^{\prime}, \mathbf{u}_{6}\right], \\
& a_{5}=\operatorname{det}\left[\operatorname{diag}\left(\mathbf{u}_{5}\right) \mathbf{u}_{6}^{\prime}, \mathbf{u}_{5}, \mathbf{u}_{6}\right], \\
& a_{6}=\operatorname{det}\left[\operatorname{diag}\left(\mathbf{u}_{5}\right) \mathbf{u}_{6}^{\prime}, \mathbf{u}_{5}^{\prime}, \operatorname{diag}\left(\mathbf{u}_{5}^{\prime}\right) \mathbf{u}_{6}\right] \text { and } \\
& a_{7}=-\operatorname{det}\left[\operatorname{diag}\left(\mathbf{u}_{5}\right) \mathbf{u}_{6}^{\prime}, \mathbf{u}_{5}, \operatorname{diag}\left(\mathbf{u}_{5}^{\prime}\right) \mathbf{u}_{6}\right] .
\end{aligned}
$$

Exactly the same analysis can be done for the seventh point in images 1 and 3, which leads to

$$
\mathbf{M}_{6 \times 6}^{\prime}\left(\mathbf{x}_{7}, \lambda_{7}, \lambda_{7}^{\prime \prime}\right)^{T}=\mathbf{0}
$$

and for the eighth point in images 2 and 3 ,

$$
\mathbf{M}_{6 \times 6}^{\prime \prime}\left(\mathbf{x}_{8}, \lambda_{8}^{\prime}, \lambda_{8}^{\prime \prime}\right)^{T}=\mathbf{0} .
$$

The vanishing of the determinants $\left|\mathbf{M}^{\prime}\right|$ and $\left|\mathbf{M}^{\prime \prime}\right|$ gives the two polynomial equations $p_{13}\left(\delta, \delta^{\prime \prime}\right)=0$ and $p_{23}\left(\delta^{\prime}, \delta^{\prime \prime}\right)=0$, where $p_{13}$ and $p_{23}$ are given as

$$
p_{13}=b_{1} \delta^{2} \delta^{\prime \prime}+b_{2} \delta \delta^{\prime \prime 2}+b_{3} \delta^{2}+b_{4} \delta \delta^{\prime \prime}+b_{5} \delta^{\prime \prime 2}+b_{6} \delta+b_{7} \delta^{\prime \prime}
$$

and

$p_{23}=c_{1} \delta^{\prime 2} \delta^{\prime \prime}+c_{2} \delta^{\prime} \delta^{\prime \prime 2}+c_{3} \delta^{\prime 2}+c_{4} \delta^{\prime} \delta^{\prime \prime}+c_{5} \delta^{\prime 2}+c_{6} \delta^{\prime}+c_{7} \delta^{\prime \prime}$.

The coefficients $b_{i}$ are polynomial expressions in $\mathbf{u}_{5}, \mathbf{u}_{5}^{\prime \prime}, \mathbf{u}_{7}$, and $\mathbf{u}_{7}^{\prime \prime}$ and the $c_{i}$ in $\mathbf{u}_{5}^{\prime}, \mathbf{u}_{5}^{\prime \prime}, \mathbf{u}_{8}^{\prime}$, and $\mathbf{u}_{8}^{\prime \prime}$, analogously to the formulas given for $a_{i}$ above.

Now, we have to solve the polynomial system of equations

$$
\left\{\begin{array}{l}
p_{12}\left(\delta, \delta^{\prime}\right)=0 \\
p_{13}\left(\delta, \delta^{\prime \prime}\right)=0 \\
p_{23}\left(\delta^{\prime}, \delta^{\prime \prime}\right)=0
\end{array}\right.
$$

for the three unknown camera parameters $\delta, \delta^{\prime}$, and $\delta^{\prime \prime}$. Since each equation is cubic in the unknowns, there may be as many as $3 \cdot 3 \cdot 3=27$ different solutions in view of Bezout's theorem, see [3]. However, as the polynomials are not dense-the monomials $\delta^{3}, \delta^{\prime 3}$ and the constant are missing in $p_{12}$-we could expect to have much less than 27 possible solutions.

Computing the resultant (see [3]), which we denote by $p_{12}^{\prime}$, of $p_{13}$ and $p_{23}$ with respect to $\delta^{\prime \prime}$ yields a polynomial equation in $\delta$ and $\delta^{\prime}$ of degree 7 with coefficients that are polynomial expressions in the image coordinates. Next, taking the resultant of the polynomials $p_{12}^{\prime}$ and $p_{12}$ with respect to $\delta^{\prime}$, one obtains a polynomial equation in $\delta$ of degree 15 .

At this stage, one might (mistakingly) conclude that there are 15 solutions. However, in the derivation, we have introduced spurious solutions, which are independent of the image data. These solutions are identified by carefully inspecting the matrix $\mathbf{M}_{6 \times 6}$ in (5). If $\delta=u_{5}$ and $\delta^{\prime}=u_{5}^{\prime}$, then the first column of the matrix $\mathbf{M}$ is a zero vector, resulting in the singularity of the matrix $\mathbf{M}$. The same is true for $\mathbf{M}^{\prime}$ and $\mathbf{M}^{\prime \prime}$ in (7) and (8), respectively. In summary, all three matrices $\mathbf{M}, \mathbf{M}^{\prime}$, and $\mathbf{M}^{\prime \prime}$ contain a zero column, hence, singular if

$$
\begin{aligned}
& \left(\delta, \delta^{\prime}, \delta^{\prime \prime}\right)=\left(u_{5}, u_{5}^{\prime}, u_{5}^{\prime \prime}\right), \\
& \left(\delta, \delta^{\prime}, \delta^{\prime \prime}\right)=\left(v_{5}, v_{5}^{\prime}, v_{5}^{\prime \prime}\right), \\
& \left(\delta, \delta^{\prime}, \delta^{\prime \prime}\right)=\left(w_{5}, w_{5}^{\prime}, w_{5}^{\prime \prime}\right),
\end{aligned}
$$

or

$$
\left(\delta, \delta^{\prime}, \delta^{\prime \prime}\right)=(0,0,0)
$$

These solutions imply, in turn, that the camera center one of the three cameras is located at

$$
(1,0,0,0),(0,1,0,0),(0,0,1,0) \text { or }(0,0,0,1) \text {. }
$$

For instance, $\delta=u_{5}$ gives the first camera center at $(1,0,0,0)$ as $\operatorname{ker}(\mathbf{P})=(1,0,0,0)$. This means that the camera center coincides with the first object point in the projective basis. These four solutions are obviously not a general situation and can be easily singled out from the final 15th degree polynomial equation. This concludes the proof that we can have at most $11=15-4$ solutions.

Remark. The special choice of coordinates can also be used to solve the minimal cases of seven points visible in two images and its Carlsson dual six points visible in three images.

\section{4 a Catalogue of Minimal Cases WITH MISSING DATA}

Having solved one minimal case with missing data, we now systematically look for all other possible cases. First, however, we can already apply Carlsson duality [2] to the problem we have solved. Recall that Carlsson and Weinshall demonstrated the following remarkable result:

Theorem 2 (see [2]). The projective reconstruction with $n$ points and $m$ images is equivalent to that with $m+4$ points and $n-4$ images. The $n$ points and $m$ images is said to be dual to $m+4$ points and $n-4$ images.

For instance, the case of seven points in two images is equivalent to six points in three images. So, knowing that six points in three images has, in general, three solutions, it follows directly by the Carlsson duality that seven points in two images also has three solutions.

Now dualizing Theorem 1 for the minimal case of eight points in three images with one point missing, we obtain another minimal case with seven points in four images where one point is missing in one of the four images. 
Corollary 1. There are, in general, 11 different solutions to the projective reconstruction for seven points in four images where one different point is missing in three of the four images.

The procedure of dualizing can also be demonstrated for the incidence relationship in (2). Start by removing the first four columns corresponding to four base points of the duality. Then, transpose the remaining matrix and add the four base columns again. After that, we obtain the following dualized incidence relation corresponding to Corollary 1 .

$$
\left[\begin{array}{ccccccc}
\times & \times & \times & \times & \times & \times & \times \\
\times & \times & \times & \times & \times & \times & 0 \\
\times & \times & \times & \times & \times & 0 & \times \\
\times & \times & \times & \times & 0 & \times & \times
\end{array}\right]
$$

\subsection{Three Images}

We can now find all other minimal cases for three images.

Let $n_{2}$ and $n_{3}$ denote the number of points which appear simultaneously in only two and three images, respectively. Clearly, $n_{3}<6$, otherwise, we have the case of (at least) six points in three images.

The number of unknowns for the three-view geometry is $3 \cdot 11$ for the three cameras and $3 \cdot\left(n_{2}+n_{3}\right)-15$ for the $n_{2}+n_{3}$ points modulo a projective transformation, while the number of equations is $4 \cdot n_{2}+6 \cdot n_{3}$. By equating the number of unknowns and the number of equations, we have necessary conditions for minimal cases,

$$
4 n_{2}+6 n_{3}=33+3\left(n_{2}+n_{3}\right)-15,
$$

i.e.,

$$
n_{2}+3 n_{3}=18 .
$$

This is a Diophantine equation whose solution is

$$
\left\{\begin{array}{l}
n_{2}=3 k \\
n_{3}=6-k
\end{array} \quad k=1, \ldots, 6\right.
$$

for $0 \leq n_{3}<6$, as $n_{3} \geq 6$ leads to the trivial nonmissing data case.

The solution $k=1$ gives $n_{2}=3$ and $n_{3}=5$ which may result in the following two incidence relations (not symmetric over three images) in addition to the one that we have solved in Theorem 1:

$$
\left[\begin{array}{cccccccc}
\times & \times & \times & \times & \times & \times & \times & \times \\
\times & \times & \times & \times & \times & \times & \times & 0 \\
\times & \times & \times & \times & \times & 0 & 0 & \times
\end{array}\right]
$$

or

$$
\left[\begin{array}{cccccccc}
\times & \times & \times & \times & \times & \times & \times & \times \\
\times & \times & \times & \times & \times & \times & \times & \times \\
\times & \times & \times & \times & \times & 0 & 0 & 0
\end{array}\right]
$$

In general, equating the number of equations and unknowns gives only necessary conditions. It is not sufficient to guarantee a finite number of solutions. For the incidence relation (12), the first two images have seven points in common, so it could be solved by Sturm's method resulting in up to three solutions. For each of these three solutions, we need to determine the geometry of the third camera. The constraint for the eighth point, visible in images one and three, is given by $p_{13}$ in (9). This is a second degree equation in the remaining unknown $\delta^{\prime \prime}$. Note that $\delta$ is already solved for using images one and two. Hence, there are up to two possibilities for the third camera. In total, there might be up to six possible solutions for this case.

For the case described by the incidence relation (13), the geometry of the first two cameras is generally uniquely determined as they have eight points in common, which is overconstrained, while there are infinitely many possibilities for the third camera, since it is underconstrained.

The other values for $k>1$ can be handled in a similar manner using resultants to eliminate the unknown variables. However, the reduced camera matrices cannot directly be used since there are not five points visible in all images. The resulting polynomial equations are of very high degree and, therefore, hard to solve. One probably needs to rely on numerical computations.

\subsection{Four Images}

Let $n_{p}, p=2,3,4$ denote the number of points which appear simultaneously in $p$ images. Equating the number of unknowns and the number of constraints gives the following Diophantine equation:

$$
4 n_{2}+6 n_{3}+8 n_{4}=44+3\left(n_{2}+n_{3}+n_{4}\right)-15
$$

i.e.,

$$
n_{2}+3 n_{3}+5 n_{4}=29 .
$$

There are many solutions to the above equation. The complete catalogue for four images consists of all solutions to the above equation. We discuss a few of the cases below for small $n_{2}$ and $n_{3}$, for which the solutions are obtainable with symbolic calculations in, for example, Maple without requiring waste computer resources.

- $n_{4}=5, n_{3}=n_{2}=1$. This is the dual of the incidence relation shown in (12),

$$
\left[\begin{array}{ccccccc}
\times & \times & \times & \times & \times & \times & \times \\
\times & \times & \times & \times & \times & \times & 0 \\
\times & \times & \times & \times & \times & \times & 0 \\
\times & \times & \times & \times & \times & 0 & \times
\end{array}\right]
$$

So, in general, there are six solutions.

- $n_{4}=5, n_{3}=0, n_{2}=4$. If the four points visible in only two images are symmetrically distributed such that no subset of seven points is common in two images, i.e.,

$$
\left[\begin{array}{ccccccccc}
\times & \times & \times & \times & \times & \times & \times & 0 & 0 \\
\times & \times & \times & \times & \times & \times & 0 & \times & 0 \\
\times & \times & \times & \times & \times & 0 & \times & 0 & \times \\
\times & \times & \times & \times & \times & 0 & 0 & \times & \times
\end{array}\right]
$$

then one can show, in the same way as in the proof of Theorem 1 , that there are up to 27 solutions! The dual case consists of five images and eight points.

- $n_{4}=4, n_{3}=3, n_{2}=0$. This is the dual of Theorem 1 whose solution is given in Corollary 1 with incidence relation (11).

The list of cases continues, but they occur less frequently in practice, and often the complexity of the involved polynomials is too high in order to solve with symbolic computations.

\section{EXPERIMENTS}

The algorithm for eight points in three images described in this paper has been implemented in Maple. We first demonstrate the 

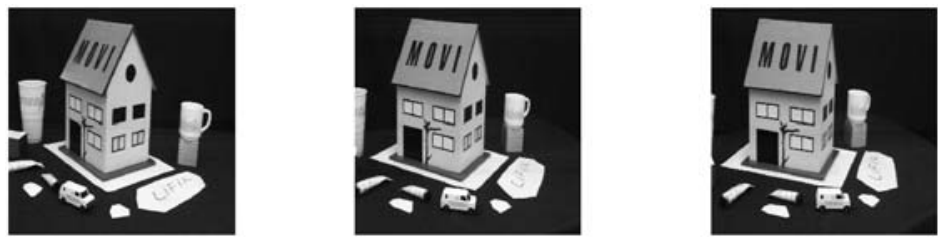

Fig. 2. The three original images used for the experiment.

algorithm in the popular sequence of images of a wooden house (cf. Fig. 2), which has been previously used for different projective reconstruction algorithms by many researchers. Three views covering about a $45^{\circ}$ rotation of the camera around the wooden house are taken. The point features are first detected as the maximum of curvatures of the B-Spline approximation of the edge chains, then automatically tracked for the three images. The location of point features is also optimized by a nonlinear subpixel corner detector. The minimal missing data is shown in Fig. 3. The first five points are visible in all three images and the sixth point is considered missing in the first, the seventh is missing in the third, and the eighth missing in the second image. Note that the seventh point is indeed missing in the third image as the side face is becoming tangent to the camera view. As more points are available in this sequence, a traditional method based on bundle adjustment has first been applied using all available 54 points. This $3 \mathrm{D}$ reconstruction is used as ground truth. The distance between the first and fifth point (which are farthest apart) is 30 units.

We start by computing the real solutions of $\delta, \delta^{\prime}$, and $\delta^{\prime \prime}$ for the projection matrices with the Maple implementation of the algorithm. For each real solution of projection matrices, we compute the projective structure of the set of eight points. The projective reconstruction is then transformed into its Euclidean representation by applying a space collineation calculated with the five known reference points from the ground truth. Finally, the root mean square (RMS) error is computed for the reconstructed sixth, seventh, and eighth point w.r.t. their known Euclidean coordinates. So, the unit for the RMS error is relative to the Euclidean 3D point positions of the ground truth.

In this real image case, very interestingly, up to the maximum of 11 real solutions are obtained! The lowest RMS solution is considered as the true solution. The other 46 matched points across the three images are also reconstructed in space for this solution. The final reconstruction of all points by the minimal missing data algorithm is superimposed with the reconstruction results obtained by a bundle adjustment. The result is illustrated in Fig. 4.

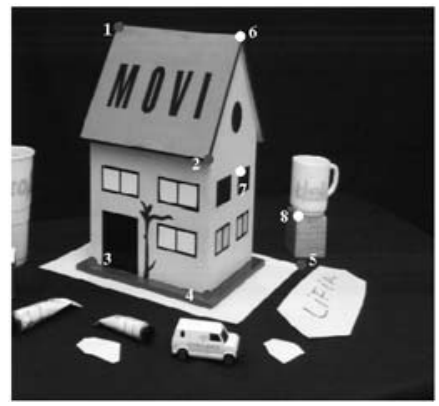

Fig. 3. The minimal missing data consisting of eight points are illustrated. The first five points are the common reference points and the last three points are the "missing" points.
The reconstruction is remarkably good, only in the side view, we can see the error is more pronounced for the farther points such as those on the cup. The readers can also compare with that presented in [14] using six points in three images.

We also check the stability of the algorithm by running on simulated data with the following set-up. We use three real camera matrices similar to the previous real image case, typically, obtained from a bundle adjustment algorithm. A set of eight known points are selected and projected by these projection matrices onto the synthesized images. Then, the projected positions of the points in the images are perturbed by varying levels of noise of a Gaussian
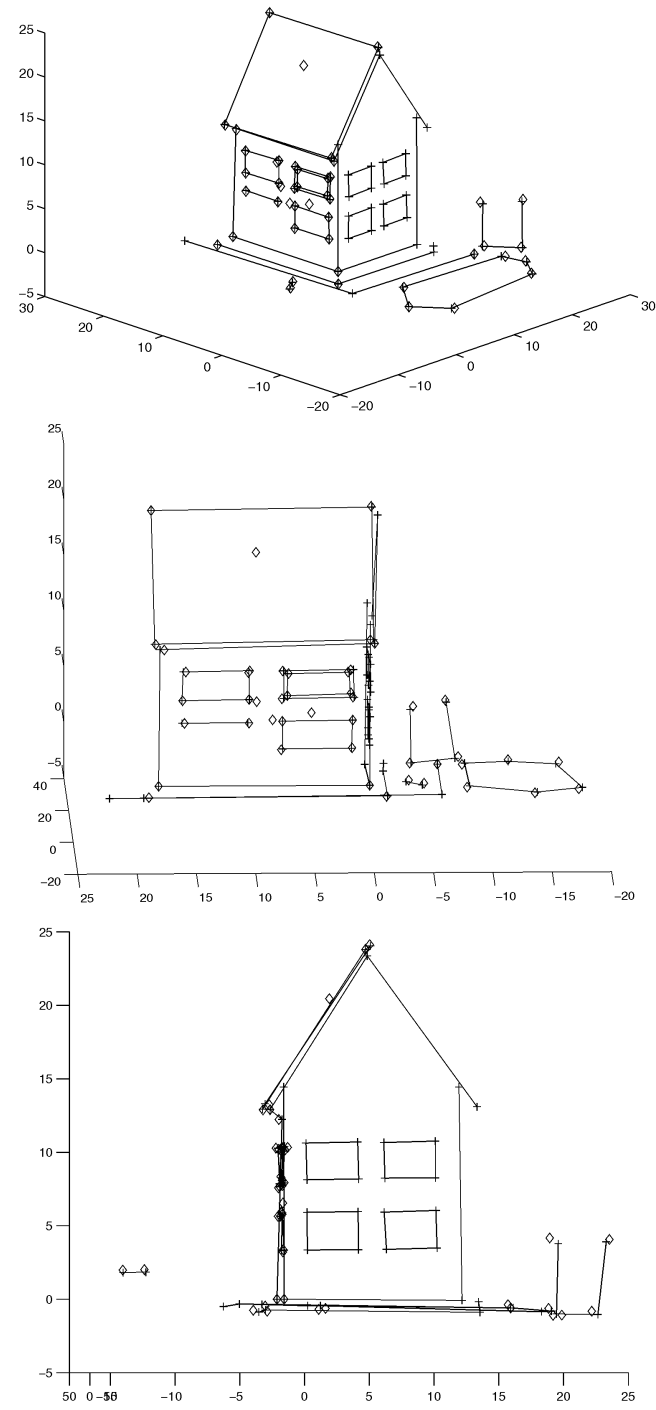

Fig. 4. A general, front and side view of two superimposed reconstructions: The points reconstructed from the minimal missing data are marked as diamonds and the points from bundle adjustment solution are marked as crosses. 


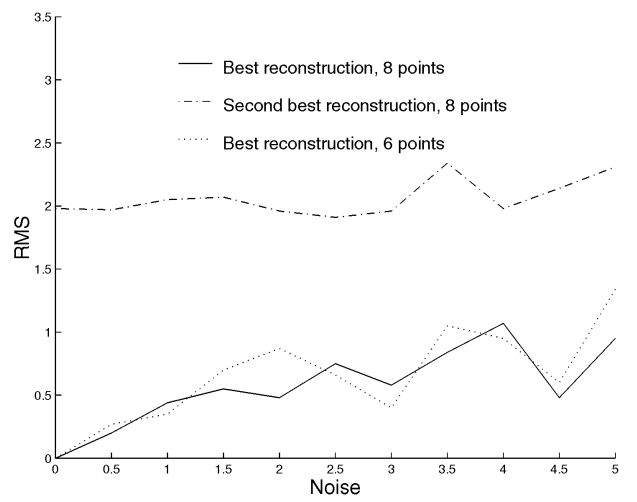

Fig. 5. RMS errors of 3D reconstruction vs. standard deviation of image noise, for the best and second best solutions of the eight point algorithm, and the best solution for the six point algorithm. The unit for the RMS error is relative to the ground truth, where the distance between the first and fifth point (which are farthest apart) is 30 units.

distribution. The realism of the simulation is preserved in this way and the image noise can be quantatively controlled as well in order to observe its influence. From the perturbed image points, the same computation procedure as for the previous real image case is performed. In addition, with the same data, the eight points were reconstructed using the six point algorithm (where all points are visible), resulting in three solutions. For each of these solutions, the seventh and eighth point were reconstructed using the obtained camera matrices. The reconstruction errors are graphed in Fig. 5, for both algorithms. In Fig. 6, the number of real solutions and the number of solutions with all points having positive depths are illustrated.

We note that the solutions degrade very smoothly with increasing noise level. The behavior of the eight point algorithm is similar to the performance of the six point algorithm. Even if there are several real solutions, we see that there are at most one solution with all points in front of the camera. It can also be seen in the experiment, there may be no positive depth solution due to the high level of noise. In other scenarios, there could possibly be several positive depth solutions. If such a case occurs, further information is necessary in order to discriminate among the solutions.

The results suggest that the algorithm presented in this paper are of practical importance even if a high degree polynomial equation has to be solved.

\section{CONCLUSIONS}

A new family of projective reconstruction problems using the minimal data from multiple uncalibrated images has been formulated and solved. Instead of the minimal data available in all images, we allow that the minimal data could be partly missing in some of the images. These minimal cases are undoubtedly of theoretical importance in order to understand the geometry of multiple views. But, also, they are of practical importance for both robust and optimal reconstruction. We first proposed a general framework to parameterize the geometry of the multiple cameras. Then, we solved the first problem of eight points in three images with one different point missing in each of the three images, proving that this problem can have as many as 11 algebraic solutions including complex and negative depth solutions. We

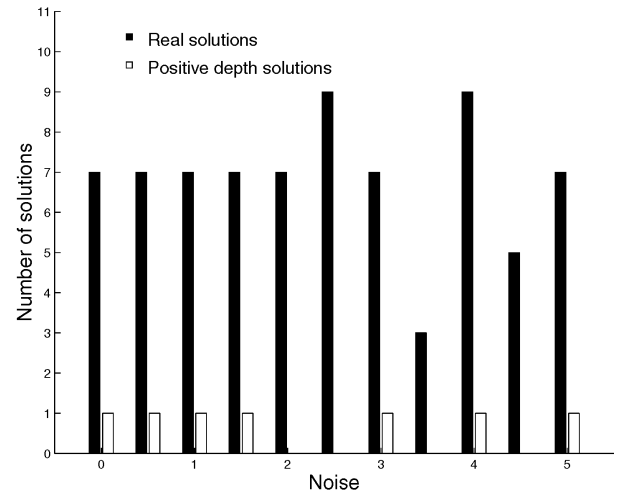

Fig. 6. The number of real solutions and solutions with all points having positive depths vs. standard deviation of image noise, for the eight point algorithm.

have also shown that the minimal cases with missing data for reconstruction is generally more complicated than those without missing data. Though we have to solve very high degree polynomial equations, the actual Maple implementation of the algorithms presented in this paper demonstrated remarkable reconstruction results. The accuracy and stability of algebraic solutions with missing data are comparable with those obtained without missing data. It suggests that these algorithms can be practically used for bootstrapping robust and optimal reconstruction. A complete catalogue of the minimal missing data cases for three and four images has also been provided.

\section{ACKNOWLEDGMENTS}

This work has been done with support from the ESPRIT LTR project CUMULI.

\section{REFERENCES}

[1] K.B. Atkinson, Close Range Photogrammetry and Machine Vision. Whittles Publishing, 1996.

[2] S. Carlsson and D. Weinshall, "Dual Computation of Projective Shape and Camera Positions from Multiple Images," Int'l J. Computer Vision, vol. 27, no. 3, pp. 227-241, 1998.

[3] D. Cox, J. Little, and D. O'Shea, Ideals, Varities, and Algorithms. New York: Springer-Verlag, 1992

[4] O.D. Faugeras, "What Can Be Seen in Three Dimensions with an Uncalibrated Stereo Rig?" Proc. European Conf. Computer Vision, pp. 563578, 1992.

[5] O.D. Faugeras and S. Maybank, "Motion from Point Matches: Multiplicity of Solutions," Technical Report 1157, INRIA, Rocquencourt, France, 1990.

[6] M.A. Fischler and R.C. Bolles, "Random Sample Consencus, A Paradigm for Model Fitting with Application to Image Analysis and Automated Cartography," Comm. Assoc. Computer Machine, vol. 24, pp. 381-395, 1981.

[7] R. Hartley, R. Gupta, and T. Chang, "Stereo from Uncalibrated Cameras," Proc. Conf. Computer Vision and Pattern Recognition, pp. 761-764, 1992.

[8] R.I. Hartley and A. Zisserman, Multiple View Geometry in Computer Vision. Cambridge Univ. Press, 2000.

[9] A. Heyden, "Geometry and Algebra of Multipe Projective Transformations," PhD thesis, Lund Inst. Technology, Sweden, 1995.

[10] A. Heyden and K. Åström, "Flexible Calibration: Minimal Cases for AutoCalibration," Int'l Conf. Computer Vision, pp. 350-355, 1999.

[11] F. Kahl and A. Heyden, "Affine Structure and Motion from Points, Lines, and Conics," Int'l J. Computer Vision, vol. 33, no. 3, pp. 163-180, 1999.

[12] Y. Ma, S. Soatto, J. Kosecká, and S. Sastry, "Euclidean Reconstruction and Reprojection up to Subgroups," Int'l J. Computer Vision, vol. 38, no. 3, pp. 219-229, 2000.

[13] S.J. Maybank and O.D. Faugeras, "A Theory of Self-Calibration of a Moving Camera," Int'l J. Computer Vision, vol. 8, no. 2, pp. 123-151, 1992.

[14] L. Quan, "Invariant of a Pair of Non-Coplanar Conics in Space: Definition, Geometric Interpretation, and Computation," Proc. Int'l Conf. Computer Vision, pp. 926-931, 1995.

[15] L. Quan, "Invariants of Six Points and Projective Reconstruction from Three Uncalibrated Images," IEEE Trans. Pattern Analysis and Machine Intelligence, vol. 17, no. 1, pp. 34-46, Jan. 1995. 
[16] A. Shashua and M. Werman, "Trilinearity of Three Perspective Views and Its Associated Tensor," Proc. Int'l Conf. Computer Vision, pp. 920-925, 1995.

[17] R. Sturm, "Das Problem der Projektivität und seine Anwendung auf dieFlächen Zweiten Grades," Math. Ann., vol. 1, pp. 533-574, 1869.

[18] C. Tomasi and T. Kanade, "Shape and Motion from Image Streams under Orthography: A Factorization Method," Int'l J. Computer Vision, vol. 9, no. 2, pp. 137-154, 1992

[19] P.H.S. Torr and A. Zisserman, "Robust Parameterization and Computation of the Trifocal Tensor," Image and Vision Computing, vol. 15, no. 8, pp. 591605, 1997.

[20] B. Triggs, "Autocalibration and the Absolute Quadric," Proc. Conf. Computer Vision and Pattern Recognition, pp. 609-614, 1997.

[21] B. Triggs, "Linear Projective Reconstruction from Matching Tensors," Image and Vision Computing, vol. 15, no. 8, pp. 617-625, 1997.

[22] Z. Zhang, R. Deriche, O.D. Faugeras, and Q.T. Luong, "A Robust Technique for Matching Two Uncalibrated Images through the Recovery of the Unknown Apipolar Geometry," technical teport, INRIA, Rocquencourt, France, 1994. 